\title{
Piece-wise variance method for signal-to-noise ratio estimation in elastic/Raman lidar signals
}

\author{
Mohd Nadzri Md Reba, Francesc Rocadenbosch, Michaël Sicard, Constantino Muñoz and Sergio Tomás \\ Universitat Politècnica de Catalunya (UPC), Dep. of Signal Theory and Communications (TSC), \\ Remote Sensing Lab (RSLAB), C/Gran Capità 1-3, D4-011, \\ 08034 Barcelona, SPAIN. \\ nadzri@tsc.upc.edu
}

\begin{abstract}
A straightforward signal-to-noise ratio (SNR) estimator for elastic/Raman lidar channels and related noise-induced errorbars is presented. The estimator is based on piece-wise estimation of the mean signal power and noise variance component under analog detection. The piece-wise estimator results are compared with those obtained from a previously published SNR parametric estimator under high and low SNR scenarios.
\end{abstract} lidar.

Keywords-Signal-to-noise ratio, variance, estimation, noise,

\section{INTRODUCTION}

SNR estimation is the determining input to assess inversion algorithm errorbars and to inter-compare system and algorithm performances [1], all of which puts research of robust SNR estimators as a priority.

Observation noise corrupting measured elastic/Raman signals at the receiver output can be of different statistical origins, namely, shot photo-induced, shot dark-current, and thermal ([2], Ap. A and [3], Ap. A). Under analog detection [4], the count-rates are high enough to assume that discrete Poisson statistics can be approximated by continuous Gaussian ones [5]. This enables to model the observation noise as rangedependent equivalent Gaussian noise.

To estimate the SNR, both the signal power and the noise standard deviation (equivalently, the noise variance) must be estimated. While a power estimate is usually derived by lowpass filtering the range-corrected observable return, derivation of the range-dependent noise variance is more involved. Here, we propose to estimate it along successive adjacent range intervals of the lidar return. This is called the piece-wise approach.

Yet, this is not the unique way to estimate the rangedependent SNR. Thus, to qualitatively assess the estimator performance, the piece-wise SNR estimator is compared with a previously published one based on parametric estimation [3].

This paper is organised as follows: Sect. II reviews SNR fundamentals and formulates the piece-wise estimator and related errorbars, Sect. III compares the proposed estimator with the parametric one and outlines the mains pros and cons along different noise-dominated elastic lidar channel examples. Conclusion remarks are given in Sect. IV.

\section{SignAL-TO-NOISE (SNR) ESTIMATION}

\section{A. SNR fundamentals}

We depart from the superposition principle by defining the background-subtracted lidar observable as

$$
z(R)=P(R)+n(R) \quad[W],
$$

where $P(R)$ is the "ideal" (i.e., noiseless) lidar power return or signal component, and $n(R)$ is the equivalent zero-mean Gaussian noise component (see Sect. I) referred to the receiver input. At his point, it is important to empathise that total noise appearing at the receiver voltage output can be referred to the optical power input by downscaling it by the system net responsivity, $R_{v}^{\prime}[\mathrm{V} / \mathrm{W}]$.

From (1) the SNR is defined as

$$
\operatorname{SNR}_{p}(R)=\frac{P(R)}{\sigma(R)} \quad \frac{[W]}{[W]},
$$

where subindex " $p$ " is a reminder of "referred to the system power input" and $\sigma(R)$ is the equivalent noise standard deviation (std. dev., for short) associated to $n(R)$.

Following [3], the equivalent noise std. dev. can be expressed as

$$
\sigma(R)=\left[a_{e q} P(R)+b_{e q}\right]^{1 / 2} \quad[W],
$$

where $a_{e q}[W]$ and $b_{e q}\left[W^{2}\right]$ are constitutive parameters of the lidar receiving channel (see (6) and Appendix A in [3] for exhaustive formulation). Thus, the term $a_{e q} P(R)\left[W^{2}\right]$ represents the shot photo-induced noise contribution modelling the inherent statistical fluctuations associated to the Poisson nature of the lidar signal received photons. The term $b_{e q}\left[W^{2}\right]$ merges into a single body shot photo-induced noise from the background component (e.g. solar radiation), shot dark-current induced noise (Poisson statistics), and thermal noise contributions (Gaussian statistics). 


\section{B. SNR Piece-wise estimation}

The piece-wise estimation procedure is based on partitioning the measurement range into adjacent intervals, $I_{j}$, of appropriate length in order to estimate both the power signal component, $P(R)$, and the noise std. dev., $\sigma(R)$, (equivalently, the noise variance) at each successive interval. Formally,

$$
\left\langle S N R_{j}\right\rangle=\frac{\hat{\mu}_{j}}{\hat{\sigma}_{j}}\left[\frac{W}{W}\right] ; \hat{\mu}_{j}=\hat{P}\left(R_{c, j}\right)[W], \quad \hat{\sigma}_{j}=\hat{\sigma}\left(R_{c, j}\right)[W], \quad R_{i} \in I_{j},
$$

where the symbols \langle\rangle and $\wedge$ mean "estimated", $R_{c, j}$ is the range corresponding to the "central" sample of interval $I_{j}$, and $\hat{\mu}_{j}$ and $\hat{\sigma}_{j}$ are the central-sample power and noise std. dev. estimates, respectively. In this work, only uniform length intervals of an odd number of samples $\left(R_{i} \in I_{j}\right), N$, are considered. At each interval $I_{j}$, the estimator is to combine $N$ samples of the lidar observable, $z(R)$, to come up with a pair of estimates $\hat{\mu}_{j}$ and $\hat{\sigma}_{j}$, namely, the mean and std. dev. of $z(R)$, respectively.

When the estimated SNR in (4) is compared with its true value,

$$
S N R_{j}=\frac{\mu_{j}}{\sigma_{j}}\left[\frac{W}{W}\right] ; \mu_{j}=P\left(R_{c, j}\right)[W], \quad \sigma_{j}=\sigma\left(R_{c, j}\right) \quad[W], \quad R_{i} \in I_{j},
$$

the estimation error is computed as the relative error in interval $I_{j}, \varepsilon_{r, j}$, defined as

$$
\left\langle S N R_{j}\right\rangle=\operatorname{SNR}_{j}\left(1 \pm \varepsilon_{r, j}\right), \quad \varepsilon_{r, j}=\left|\frac{\sigma_{\hat{\mu}_{j}}}{\hat{\mu}_{j}}\right|+\left|\frac{\sigma_{s_{j}}}{\hat{\sigma}_{j}}\right|, \quad R \in I_{j},
$$

where $\sigma_{\hat{\mu}_{j}}$ and $\sigma_{s_{j}}$ are respectively the estimation errors associated to the signal component estimate, $\hat{\mu}_{j}$, and to the noise std. dev. estimate, $\sigma_{j}$. Estimation of these quantities is discussed next (Sects. II.C-D). In turn, the ratios $\left|\sigma_{\hat{\mu}_{j}} / \hat{\mu}_{j}\right|$ and $\left|\sigma_{s_{j}} / \hat{\sigma}_{j}\right|$ respectively express the relative estimation errors associated to $\hat{\mu}_{j}$ and $\hat{\sigma}_{j}$.

\section{Signal component estimation}

The estimation is performed in two steps: First, the mean component, $\hat{\mu}(R)$, is derived by low-pass filtering (equivalently, by range smoothing) the observable lidar return, $z(R)$, with a window length equal to the interval length, $N$. This ensures that all available samples from $I_{j}$ are used to estimate the central one. In fact, filtering the range-corrected observable with just a rectangular filter and correcting by $R^{2}$ afterwards is the preferred method to reduce distortion. Second, the centralsample signal component estimate is retained so that

$$
\mu_{j}=\hat{P}\left(R_{c, j}\right)=\left.Z_{L P}(R)\right|_{R=R_{c, j}} .
$$

When a rectangular window is used, which represents estimating the mean of the samples in $I_{j}$, the error std. dev. of $\mu_{j}$ estimate is given by [6]

$$
\sigma_{\mathfrak{\mu}_{j}}=\frac{\sigma_{j}}{\sqrt{N}} .
$$

To compute the unknown $\sigma_{j}$, we use its estimate, $\sigma_{j} \approx \hat{\sigma}_{j}$, instead. This is discussed next.

\section{Noise variance estimation}

To piece-wise estimate the noise variance at each interval $I_{j}$, an estimate of the observation noise, $n(R)$ in (1), is first computed as

$$
\hat{n}(R)=z(R)-\hat{\mu}(R) .
$$

Note that this is equivalent to high-pass filtering the lidar observable $z(R), \hat{n}(R)=Z_{H P}(R)$.

The central-sample noise std. dev. is directly computed from the unbiased variance of $n(R)$ as

$$
\hat{\sigma}_{j}=\sqrt{\hat{V}[\hat{n}(R)]} \frac{1}{\sqrt{1-1 / N}}, \quad R \in I_{j},
$$

where the term $\frac{1}{\sqrt{1-1 / N}}$ is a (rectangular window) correction factor that takes into account the fact that the noise variance is computed at the high pass filter output, when in fact, it must be estimated at its input [7].

The estimation error associated to (10) (unbiased std. dev. estimation of a sample of $N$ data values) is given by [6]

$$
\sigma_{s_{j}} \approx \frac{\sigma_{j}}{\sqrt{2(N-1)}} .
$$

\section{DISCUSSION}

\section{A. Case example and limitations}

Figs. 1-2 illustrate operation of the SNR piece-wise estimator and the impact different choices of the (rectangular) low-pass filter window length (Sect. II.C), $N$, have on the final SNR estimate.

Two different spatial resolutions have been considered: A "high resolution" case, with $N=7$ samples, and a "low resolution" case, with variable $N$ (21 samples from $0.37 \mathrm{~km}$ $\left(R_{\text {min }}\right)$ up to $1.90 \mathrm{~km}, 41$ samples up to $6.31 \mathrm{~km}$, and 71 samples up to $\left.9.01 \mathrm{~km}\left(R_{\max }\right)\right)$. In both cases, the raw resolution is 7.5 $\mathrm{m}$, which translates into resolutions of $150 \mathrm{~m}$ and [150 300 525] m, respectively. The "interval no." notation corresponds to the following "central-sample" ranges: $I_{1}\left(R_{c, 1}=1 \mathrm{~km}\right), I_{2}\left(R_{c, 2}=3 \mathrm{~km}\right), I_{3}\left(R_{c, 3}=5 \mathrm{~km}\right), I_{4}\left(R_{c, 4}=7 \mathrm{~km}\right)$, and $I_{5}\left(R_{c, 5}=8.5 \mathrm{~km}\right)$.

As seen from Fig. 2 and Fig. 1b, $\hat{\mu}_{j}$ is the least sensitive estimate to the chosen resolution (Fig. 2a) while this is not the case for $\hat{\sigma}_{j}$ and SNR estimates (intervals $I_{1}-I_{3}$ in Fig. 2b). 
This is because in the "low resolution" case, the final value of the noise std. dev., $\hat{\sigma}_{j}$, is erroneously increased by unwanted leakage of the signal component at the high-pass filter output. This leakage is evidenced by noise spikes in Fig. 2c (intervals $I_{1}-I_{3}$ ) and Fig. 2d (interval $I_{1}$ only) and corresponds to fast signal transients of the lidar signal (Fig. 1a) at the end of the boundary layer $(R \approx 1.5 \mathrm{~km})$ and aerosol layers $(3-4 \mathrm{~km}$ and 5 $5.5 \mathrm{~km}$ ranges).

Resolution-induced errors are linked to the shape of the signal rather and hence cannot be estimated from the noiseinduced errorbars of (8),(11). They can only be minimised by decreasing the interval length, $N$, which imposes a trade-off with the general requirement of low noise-induced SNR errorbars ((6), (8) (11)), for they decrease by increasing $N$.

\section{B. Comparison with parametric estimation}

The parametric estimation approach [3] uses the noise model of (3) and a noise realization estimate, $\hat{n}(R)$ (9), to find the best system noise parameters, $\bar{v}_{e q}=\left(a_{e q}, b_{e q}\right)$-the so-called noise state vector-, under a minimum least-squared error criterion,

$$
\left.\min \left\{\left\|\left(\hat{n}(R)^{2}-\left[a_{e q} P(R)+b_{e q}\right]\right) \cdot w(R)\right\|^{2}\right\}\right|_{e q}, b_{e q},
$$

where $w(R)$ is the weighting function and the rest of variables have already been defined in (3) and (9) above. An initial guess of $\bar{v}_{e q}$ is required to solve (12).

The second simulation example considers a 532-nm elastic lidar signal synthesized by means of a homogeneous aerosol extinction of $\alpha_{\text {aer }}=0.1 \mathrm{~km}^{-1}$ and a constant lidar ratio of $25 \mathrm{sr}$ in the mixing layer $(0.2-3 \mathrm{~km})$, and a US-standard atmosphere $\left(\mathrm{T}=-2^{\circ} \mathrm{C}, \mathrm{P}=1025 \mathrm{hPa}\right.$ ) to model molecular components. Two cases are considered to compare the piece-wise and the parametric SNR estimator: Case 1 "low SNR" corresponding to a SNR in the $10^{2}-1$ range (Figs.3, 5, 6a), and case 2 "high SNR", SNR in the $10^{4}-10^{2}$ range (Figs.4, 6b). See [3] for details of similar Figs. 3-4.

From Fig. 6, it is seen both estimators perform best under low SNRs (case 1). Thus, for high SNRs, the piece-wise estimator is more sensitive to fast transients of the lidar signal (boundary layer discontinuity at $\mathrm{R}=3 \mathrm{~km}$ ) causing resolution errors to prevail while the parametric estimator fails to estimate the $b_{e q}$ noise-state component (12), usually under shot photoinduced noise-limited situations (this is evidenced by an error estimation offset in the $3-4 \mathrm{~km}$ range of Fig. $6 \mathrm{~b}$ ). The fact that both estimators use an estimate of the observable noise, $\hat{n}(R)$ in (9), as the primary quantity from which either the variance (10) or the noise state-vector is derived (12) justifies this similar behaviour.

Finally, a main drawback of parametric estimator is the need for a relatively accurate convergent initial guess.

\section{CONCLUSIONS AND FUTURE WORK}

A piece SNR estimator for elastic/Raman lidar channels has been formulated along with noise-induced errorbars. A tradeoff arises between the selected spatial resolution and the noiseinduced estimation error for the range-dependent variance estimate is very sensitive to resolution errors caused by fast transients of the signal. Selection of the optimal interval length is still a matter of research but even fixed resolutions of hundreds of meters enable practical estimations for tropospheric systems.

\section{ACKNOWLEDGMENT}

The authors wish to acknowledge the following entities for partially supporting this research work and lidar systems developed at UPC: European Union and FEDER funds under the EARLINET-ASOS (EU Coordination Action) contract $\mathrm{n}^{\circ}$ 025991 (RICA), and (EU Specific Support Action) contract $n^{\circ}$ 011863 (RIDS): "Technology development programme towards a European Extremely Large Telescope"; MCYT (Spanish Ministry of Science and Technology) and FEDER funds under the projects TEC2006-07850/TCM and REN200309753-C02-02, Complementary Actions CGL2006-26149E/CLI, CTM2006-27154-E/TECNO, and Special Action REN2002-12784-E; MITYC (Spanish Ministry of Industry, Tourism and Commerce) under the PROFIT project, CIT020400-2005-56. MCYT is also thanked for the Ramón y Cajal position hold by Dr. M. Sicard, and Local Government of Catalonia (Generalitat de Catalunya/AGAUR) for Mr. Md. Reba's predoctoral fellowship.

\section{REFERENCES}

[1] F. Rocadenbosch, A. Comerón, M. Sicard, M. N. Md Reba, "Statistical considerations on the extinction error variance for the Raman lidar inversion algorithm," Proc. IEEE International Geoscience and Remote Sensing Symposium (IGARSS-07), in press.

[2] F. Rocadenbosch, A. Comerón and D. Pineda, "Assessment of lidar inversion errors for homogeneous atmospheres," Appl. Opt. 37 (12), 2199-2206 (1998).

[3] M. N. Md Reba, F. Rocadenbosch, M. Sicard, "A straightforward signalto-noise ratio estimator for elastic/Raman lidar signals," SPIE Proc. 6362, pp. 626223-1/626223-12, 2006 [12th International Symposium on Remote Sensing, Conf. on Remote Sensing of Clouds and the Atmosphere XI, Stockholm (Sweden), 11-14 Sept. 2006)].

[4] "Photon Counting using Photomultiplier Tubes," Hamamatsu Photonics, AN-TPHO9001E02, May 1998 (Japan 2000).

[5] R.J. Barlow, "Theoretical Distributions", Chap.3 in Statistics: A Guide To The Use Of Statistical Methods In The Physical Sciences, F. Mandl, R.J. Ellison, D.J. Sandiford, Eds., (Wiley, Chichester, England, 1989), pp. $20-47$.

[6] R.J. Barlow, "Estimation", Chap. 5 in Statistics: A Guide To The Use Of Statistical Methods In The Physical Sciences, F. Mandl, R.J. Ellison, D.J. Sandiford, Eds., (Wiley, Chichester, England, 1989).

[7] A.B. Carlson, "Signal transmission and filtering," Chap. 3 in Communication Systems, 3rd ed. (McGraw-Hill, Singapore, 1986). 

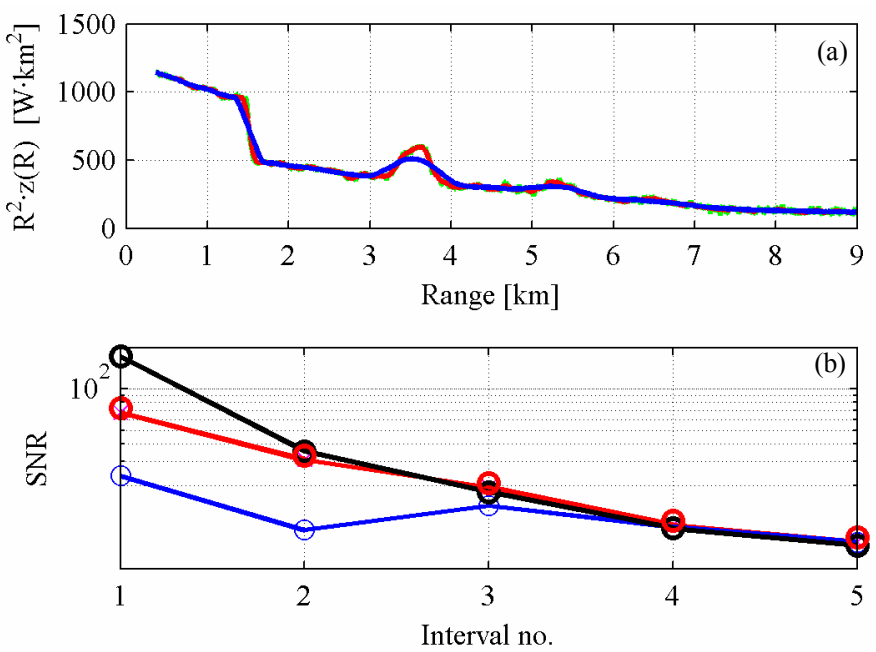

Figure 1. (a) Range-corrected 532-nm elastic return, (red/blue) high/low spatial resolution mean estimate, (7). (b) SNR estimate, (black) true SNR, (red/blue) related high/low-resolution SNR estimates, (6).
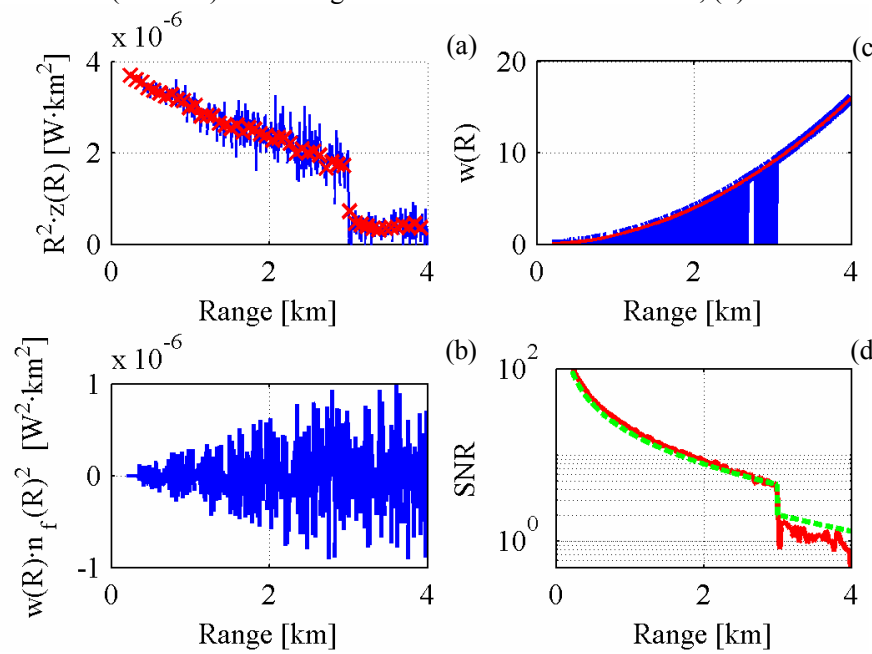

Figure 3. 532-nm elastic lidar return and SNR parametric estimation (case 1, $\left.\mathrm{SNR}\left(\mathrm{R}_{\min }\right)=10^{2}\right)$. (a) Range-corrected noisy observable, (b) weighted filtered noise, (c) weight function, (d) SNR estimate (red) vs. true SNR (green).

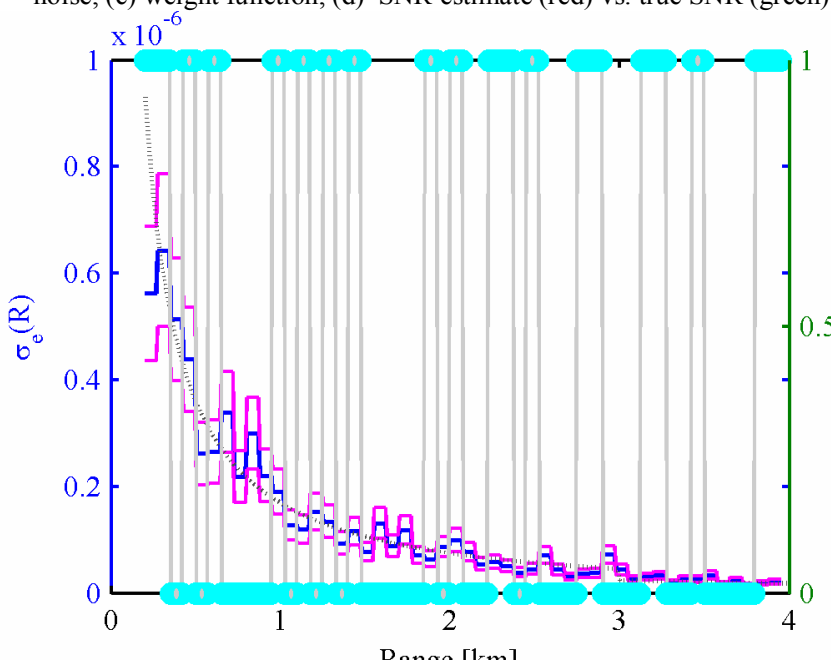

Figure 5. (Case 1) Noise standard deviation (std. dev.) piece-wise estimation. (blue) SNR estimate, (magenta) errorbars, (dotted grey) true std. dev.
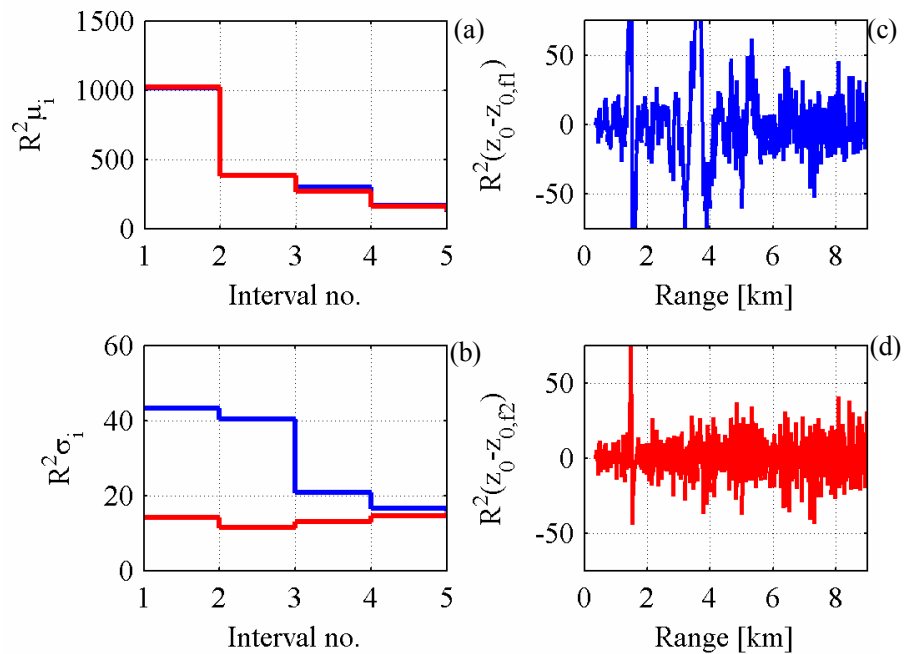

Figure 2. Resolution-induced errors. (a) Range-corrected mean, (7), and (b) std. dev. estimates, (10) (high/low resolution in red/blue trace). (c)(d) Highpass noise estimates (9) using low/high resolution, respectively.
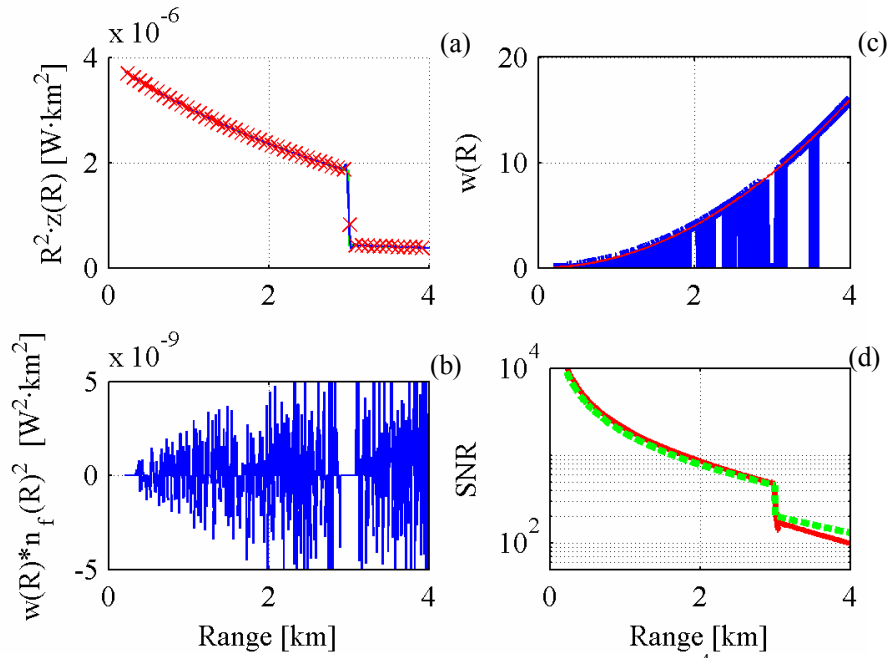

Figure 4. Same as Fig. 1 for case 2, $\operatorname{SNR}\left(\mathrm{R}_{\min }\right)=10^{4}$.

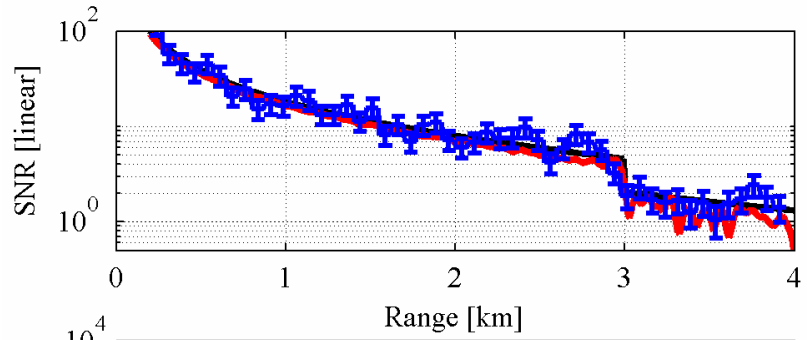

(a)

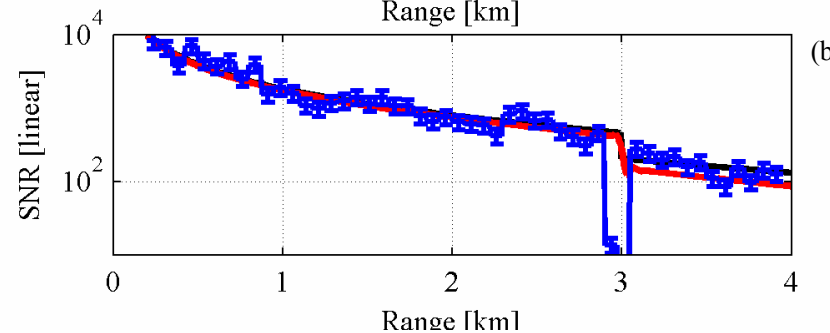

Figure 6. Comparison between the piece-wise (solid blue) and the parametric (red) SNR estimators. True SNR (black). (a) case 1, (b) case 2. 